\title{
Lung Ultrasonography Versus Chest X Ray for Diagnosing Pneumonia in Children with Fever and Respiratory Distress: A Prospective Blind Study
}

\author{
Rania Salah El Zayat ${ }^{1, ~ *, ~ W a e l ~ A b b a s s ~ B a h b a h ~}{ }^{1}$, Waleed Abd Elfattah Mousa ${ }^{2}$ \\ ${ }^{1}$ Department of Pediatrics, Faculty of Medicine, Menoufia University, Menoufia, Egypt \\ ${ }^{2}$ Department of Radiodiagnosis, Faculty of Medicine, Menoufia University, Menoufia, Egypt \\ Email address: \\ raniaelzayat1@yahoo.com(R. S. ElZayat),wael_bahbah@yahoo.com(W.A. Bahbah), waleedmousa75@gmail.com(W.A.E. Mousa) \\ ${ }^{*}$ Corresponding author
}

\section{To cite this article:}

Rania Salah El Zayat, Wael Abbass Bahbah, Waleed Abd Elfattah Mousa. Lung Ultrasonography Versus Chest X Ray for Diagnosing Pneumonia in Children with Fever and Respiratory Distress: A Prospective Blind Study. American Journal of Pediatrics. Vol. 4, No. 1, 2018, pp. 15-20. doi: 10.11648/j.ajp.20180401.14

Received: February 24, 2018; Accepted: March 21, 2018; Published: April 14, 2018

\begin{abstract}
Pneumonia is a major cause of morbidity and mortality in children especially in developing countaries. Lung ultrasonography (LUS) is an accepted safe diagnostic tool with many advantages over chest x-ray (CXR). The objective of this prospective observational study was to evaluate the usefulness of LUS in comparison with CXR for diagnosing pneumonia in 60 children presented with fever and respiratory distress. For each patient complete diagnostic workup was performed including history, physical examination, routine laboratory investigations, CXR and LUS. LUS was performed by a sonographer blinded to patient's clinical and CXR findings. The patients were classified into; Pneumonia group: included 45 patients diagnosed by two clinicians as having community acquired pneumonia (CAP) based on the recommendations of British Thoracic Society and Non pneumonia group: included 15 patients received other diagnoses. The clinicians were blinded to CXR and LUS findings. In pneumonia group, sonographic and radiographic findings compatible with pneumonia were found in $95.6 \%$ and $88.9 \%$ of patients respectively. In non pneumonia group, LUS can exclude pneumonia in $93.3 \%$ of patients while CXR excluded pneumonia in $86.7 \%$ of them. The sensitivity, specificity, positive predictive value, negative predictive value and accuracy of LUS to demonstrate lung involvement were higher than those of CXR $(95.6 \%, 93.3 \%, 97.7 \%, 85.7 \%$ and $94.5 \%$ versus $88.9 \%, 86.7 \%, 95.2 \%, 72.2 \%$ and $86.7 \%$ respectively). LUS was superior to CXR in identifying pleural effusion and multiple consolidation in more than one lobe. Being safer, cheaper and relatively more time saving, lung ultrasonography offers an important contribution to the diagnosis of CAP in febrile children with respiratory distress compared to chest X-ray.
\end{abstract}

Keywords: Lung Ultrasonography, Chest X-ray, Pneumonia, Community Acquired Pneumonia, Respiratory Distress

\section{Introduction}

According to the World Health Organization (WHO), Pneumonia accounts for $16 \%$ of all deaths of children under 5 years old, killing about 920.000 children in 2015 [1]. Africa is the region with the second highest estimate of clinical pneumonia incidence in children less than 5 years $(0.33$ episodes per child-year) coming closely after South-East Asia (0.36 episodes per child-year) [2]. In Egypt, it was estimated that $10 \%$ of children deaths below the age of 5 years is caused by pneumonia and other acute respiratory infections [3].
Early and correct diagnosis is strongly required for proper management of pneumonia. There is still a debate about the usefulness of imaging techniques in the diagnosis of CAP. Although several guidelines $[4,5]$ do not recommend the use CXR when diagnosing mild uncomplicated pneumonia in children, it is actually frequently performed to confirm the diagnosis of CAP [6] and absence of CXR confirmation leads overestimation of the incidence of CAP and unnecessary overuse of antibiotics [7].

The use CXR has many disadvantages, including the risk of exposure to ionizing radiation with its subsequent hazards 
[8], difficulty in scanning of about $40 \%$ of lung field though a single projection (which is usually hidden by overlying cardiac, mediastinal and subdiaphragmatic structures) [9] and low sensitivity when compared with computerized tomography $(\mathrm{CT})$ scan $[10,11]$. In countries with limited resources like Egypt other disadvantages are the unavailability and lack of portability. Even when CXR is available, there is a practical time delay for obtaining and interpreting the images [12]

Lung ultrasonography seems to be an attractive alternative to the standard CXR being safe, portable, inexpensive, repeatable and relatively time saving [12]. Also in contrast to CXR setting, parents can be allowed to attend LUS scanning to support and calm the child [13] especially in such a situation in which sedation may have deleterious effects.

The aim of this work was to evaluate the usefulness of lung ultrasonography (LUS) in comparison with chest $\mathrm{x}$-ray (CXR) for diagnosing pneumonia in children with fever and respiratory distress.

\section{Methods}

\subsection{Study Design, Settings \& Population}

This prospective cohort study was conducted at Pediatric Department of Menoufia university hospital, Egypt, between October 2016 and March 2017. Inclusion criteria were (1) Age between 2 and 60 months, (2) Presence of fever $>38.5^{\circ} \mathrm{C}$ together with increased respiratory rate more than expected for age $>50$ breaths/ min for those less than 12 months and $>$ 40 breaths/min for patients between 1 and 5 years) with or without other signs of respiratory distress (retractions whether suprasternal, subcostal or intercostal, nasal flaring, grunting or pulse oximetry $<90 \%$ on room air) [5], (3) patient was previously healthy before the previous complaints, (4) the study sonographer is available for LUS during patient evaluation. Patients with co-existing chronic lung disease (e.g. bronchial asthma, cystic fibrosis, bronchiectasis...etc), with cardiac, lung or airway congenital anomalies, receiving antibiotic treatment for any reason in the preceding month, who were hemodynamically unstable and those whose parents refuse to perform CXR or LUS were excluded.

\subsection{Interventions and Data Collection}

For each eligible patient complete diagnostic workup was performed on admission including thorough history, physical examination, LUS, CXR, routine laboratory investigations (complete blood count and $\mathrm{C}$ reactive protein) and other laboratory tests if clinically warranted. After first evaluation, enrolled patients underwent LUS then CXR and blood tests. Patients were assessed by two expert clinicians separately for having community acquired pneumonia based on the criteria of British Thoracic Society for diagnosing pneumonia in children [4]. The two clinicians were blinded to CXR and LUS findings. Then the patients were classified into two groups:

1. Pneumonia group: including patients diagnosed as having CAP. The patient was enrolled in this group only when both clinicians report the same diagnosis of community acquired pneumonia.

2. Non pneumonia group: including patients who received another diagnosis.

\subsection{Imaging Studies}

Postero-anterior chest radiographs were done in supine or sitting position as recommended in recently published guidelines [4] and were analyzed by the radiologist on duty who is blinded to LUS findings and then interpreted by an expert radiologist after hiding the patient's data. Chest x-ray was considered positive when there was primary end point consolidation and/or primary end point pleural effusion in accordance with the WHO criteria for the standardized interpretation of pediatric chest radiographs for the diagnosis of pneumonia [14].

Lung sonographic examination was performed by an expert radiologist using Toshiba XARio utrasound device with 3-5 MHz convex and 5-12 MHz linear array probes. The probes were placed in the anterior, lateral and posterior thoracic regions over the intercostal spaces in a perpendicular, oblique and transverse direction where the images were clearest. To scan the posterior thorax lateral decubitus and sitting positions were used. Lung ultrasound was considered positive on the finding of a lung consolidation (appears as subpleural echo-poor region or one with tissue-like echotexture) with evidence of sonographic air bronchograms [15]. The presence of pleural effusion was also evaluated. Sonographic findings were classified into specific patterns based on the literature [16-19].

\subsection{Ethical Considerations}

All procedures performed during the study were in accordance with the ethical standards of Menoufia University Institutional Research Committee. Informed consent was obtained from the guardian of each participant included in the study.

\subsection{Data Management and Analysis}

Results were tabulated and statistically analyzed by using a personal computer using SPSS v. 22 (IBM Corp Chicago, IL, USA). The Kolmogorov- Smirnov, Shapiro and D'agstino tests were used to verify the normality of distribution of variables. Categorical variables were compared using Chi-square test or Fisher exact test. Student t-test was used to compare two groups for normally distributed quantitative variables while Mann Whitney test was used to compare two groups for abnormally distributed quantitative variables. The diagnostic performance of LUS was assessed by traditional parameters (sensitivity, specificity, positive and negative predictive value, accuracy) as described elsewhere [20]. Significance of the obtained results was judged at the $5 \%$ level.

\section{Results}

One hundred and two children with fever and respiratory 
distress were initially evaluated. Thirty nine children met exclusion criteria and other three children were excluded as their parents refused to participate in the study. Of the sixty eligible patients, forty five patients ( 29 male and 16 female) were finally diagnosed clinically as having community acquired pneumonia (mean age 24.11 \pm 9.42 months). The other fifteen patients were diagnosed with other diseases (10 with acute bronchiolitis, 4 with acute bronchitis and 1 with pulmonary oedema).

The demographic, clinical and laboratory characteristics of patients were demonstrated in [table 1]. There was no statistically significant difference between pneumonia and non-pneumonia groups regarding age, sex, weight, length and respiratory rate. As regards to signs of respiratory distress, only chest indrawing was significantly more prevalent in pneumonia group. Abnormal auscultatory findings were heard in all pneumonia cases \& in 14/15 non-pneumonia cases. Wheezes were significantly more frequent in nonpneumonia group while bronchial breathing was heard only in 9 patients with pneumonia. Neutrophil percent \& Creactive protein was significantly higher in patients with pneumonia compared to those without pneumonia $(\mathrm{P}=0.041$ and $<0.001$ respectively). However no statistically significant difference was found between groups regarding hemoglobin level, total leukocyte count, lymphocytes or platelet count.

Table 1. Demographic, clinical and laboratory data of studied patients.

\begin{tabular}{llll}
\hline Data & Pneumonia $(\mathbf{n}=\mathbf{4 5})$ & Non-pneumonia $(\mathbf{n}=\mathbf{1 5})$ & P-value \\
\hline Age (months) & $24.11 \pm 9.42$ & $20.67 \pm 4.43$ & 0.179 \\
Sex & & & \\
Female & $16(35.6 \%)$ & $5(33.3 \%)$ & 0.876 \\
Male & $29(64.4 \%)$ & $10(66.7 \%)$ & 0.169 \\
Weight (kg) & $12.66 \pm 2.13$ & $11.80 \pm 1.82$ & 0.192 \\
Length(cm) & $82.04 \pm 8.02$ & $78.40 \pm 7.65$ & 0.214 \\
Respiratory rate (Bpm) & $60.47 \pm 9.34$ & $63.93 \pm 8.94$ & 0.406 \\
Cough & $44(97.8 \%)$ & $14(93.3 \%)$ & $<0.001$ \\
Chest indrawing & $42(93.3 \%)$ & $7(46.7 \%)$ & 0.443 \\
Nasal flaring & $29(64.4 \%)$ & $8(53.3 \%)$ & 0.527 \\
Grunting & $14(31.1 \%)$ & $6(40 \%)$ & 0.732 \\
Oxygen saturation $<90 \%$ & $2(4.4 \%)$ & $1(6.6 \%)$ & 0.080 \\
Abnormal auscultatory findings & $45(100 \%)$ & $14(93.3 \%)$ & 0.096 \\
Crepitations & $29(64.4 \%)$ & $6(40 \%)$ & $<0.001$ \\
Wheezes & $3(6.6 \%)$ & $11(73.3 \%)$ & 0.083 \\
Sonorus rhonchi & $6(13.3 \%)$ & $5(33.3 \%)$ & 0.389 \\
Diminished breath sounds & $35(77.8 \%)$ & $10(66.7 \%)$ & 0.060 \\
Bronchial breathing & $9(20 \%)$ & $0(0 \%)$ & 0.216 \\
Hb (gm\%) & $11.08 \pm 1.70$ & $10.48 \pm 1.30$ & 0.494 \\
TLC (10 $/ \mathrm{L})$ & $13.53 \pm 4.18$ & $12.67 \pm 4.29$ & 0.041 \\
Neutrophil (\%) & $61.00 \pm 9.51$ & $55.13 \pm 9.20$ & 0.844 \\
Lymphocytes (\%) & $30.69 \pm 9.79$ & $30.13 \pm 8.16$ & 0.595 \\
Platelets (x10/L) & $212.24 \pm 95.97$ & $228.33 \pm 115.15$ & $<0.001$ \\
Positive CRP & $41(91.1 \%)$ & $7(46.7 \%)$ & \\
\hline
\end{tabular}

Bpm: breaths/minute; Hb: Haemoglobin; TLC: Total leukocyte count ; CRP: C-reactive protein. Data are presented in numbers (\%) or as mean $\pm \mathrm{SD}$.

Among 45 patients with CAP, lung ultrasound findings were compatible with pneumonia in 43 patients and while chest X-ray findings were compatible with pneumonia in 40 patients. On the other hand 2 patients had a negative LUS and 5 had a negative CXR [table 2]. Sonographic findings were subpleural consolidation and dynamic air bronchograms in 43 patients, fluid bronchgrams in 14 patients, multiple $\mathrm{B}$ lines in 22 patients, pleural line irregularities in 8 patients and pleural effusion in 11 patients [figure 1]. Primary end point consolidation was detected by CXR in 40 patients, associated with pleural effusion in 7 of them. Ultrasonography was more superior to CXR in identifying consolidation in more than one lobe where the difference between the two diagnostic tools was statistically significant $(\mathrm{P}=0.048)$ [table 3].

In non pneumonia group LUS can exclude pneumonia in 14/15 patients while CXR excluded pneumonia in 13/14 of them. There was one sonographic false-positive result. It was for a child affected with acute bronchiolitis in which LUS revealed a small subpleural consolidation with pleural line irregularities. There were two radiographic false-positive results. They were also for 2 patients, one diagnosed with acute bronchiolitis and the other with mild pulmonary oedema and both show areas of basal consolidation [tables 2, 3].

Table 2. Comparison between lung ultrasonography and chest $x$-ray results in patients with pneumonia and without pneumonia.

\begin{tabular}{|c|c|c|c|c|c|c|}
\hline & \multicolumn{2}{|l|}{ Pneumonia } & \multirow{2}{*}{ Total } & \multicolumn{2}{|c|}{ Non-pneumonia } & \multirow{2}{*}{ Total } \\
\hline & CXR negative & CXR positive & & CXR negative & CXR positive & \\
\hline LUS negative & 0 & 2 & 2 & 12 & 2 & 14 \\
\hline LUS positive & 5 & 38 & 43 & 1 & 0 & 1 \\
\hline Total & 5 & 40 & 45 & 13 & 2 & 15 \\
\hline
\end{tabular}

CXR: Chest X ray; LUS: Lung Ultrasonography. 
Table 3. Chest $x$-ray and lung ultrasound findings in studied patients.

\begin{tabular}{llll}
\hline Finding & $\begin{array}{l}\text { Pneumonia } \\
\text { (n= 45) }\end{array}$ & $\begin{array}{l}\text { Non-pneumonia } \\
\text { (n= 15) }\end{array}$ & P-value \\
\hline - CXR findings & & & $2(13.3 \%)$ \\
Primary end point consolidation & $40(88.9 \%)$ & $1(6.6 \%)$ \\
$\quad$ Confined to one lobe & $37(82.2 \%)$ & $1(6.6 \%)$ & $<0.001$ \\
$\quad$ In more than one lobe & $3(6.7 \%)$ & $0(0.0 \%)$ & 0.104 \\
Primary end point pleural effusion & $7(15.6 \%)$ & $1(6.7 \%)$ & $<0.001$ \\
- LUS findings & & $1(6.7 \%)$ & \\
Consolidation & $43(95.5 \%)$ & 0 & \\
$\quad$ Confined to one lobe & $33(73.3 \%)$ & $0(0.0 \%)$ & 0.001 \\
In more than one lobe & $10(22.2 \%)$ & $0(0.0 \%)$ & 0.014 \\
Dynamic Air Bronchogram & $43(95.6 \%)$ & $0(0.0 \%)$ & 0.001 \\
Fluid Bronchogram & $14(31.1 \%)$ & $1(6.7 \%)$ & 0.297 \\
Multiple B lines & $22(48.9 \%)$ & $0(0.0 \%)$ & 0.034 \\
Pleural line irregularities & $8(17.8 \%)$ & & \\
Pleural effusion & $11(24.4 \%)$ & & \\
\hline
\end{tabular}

CXR: Chest X ray; LUS: Lung Ultrasonography.

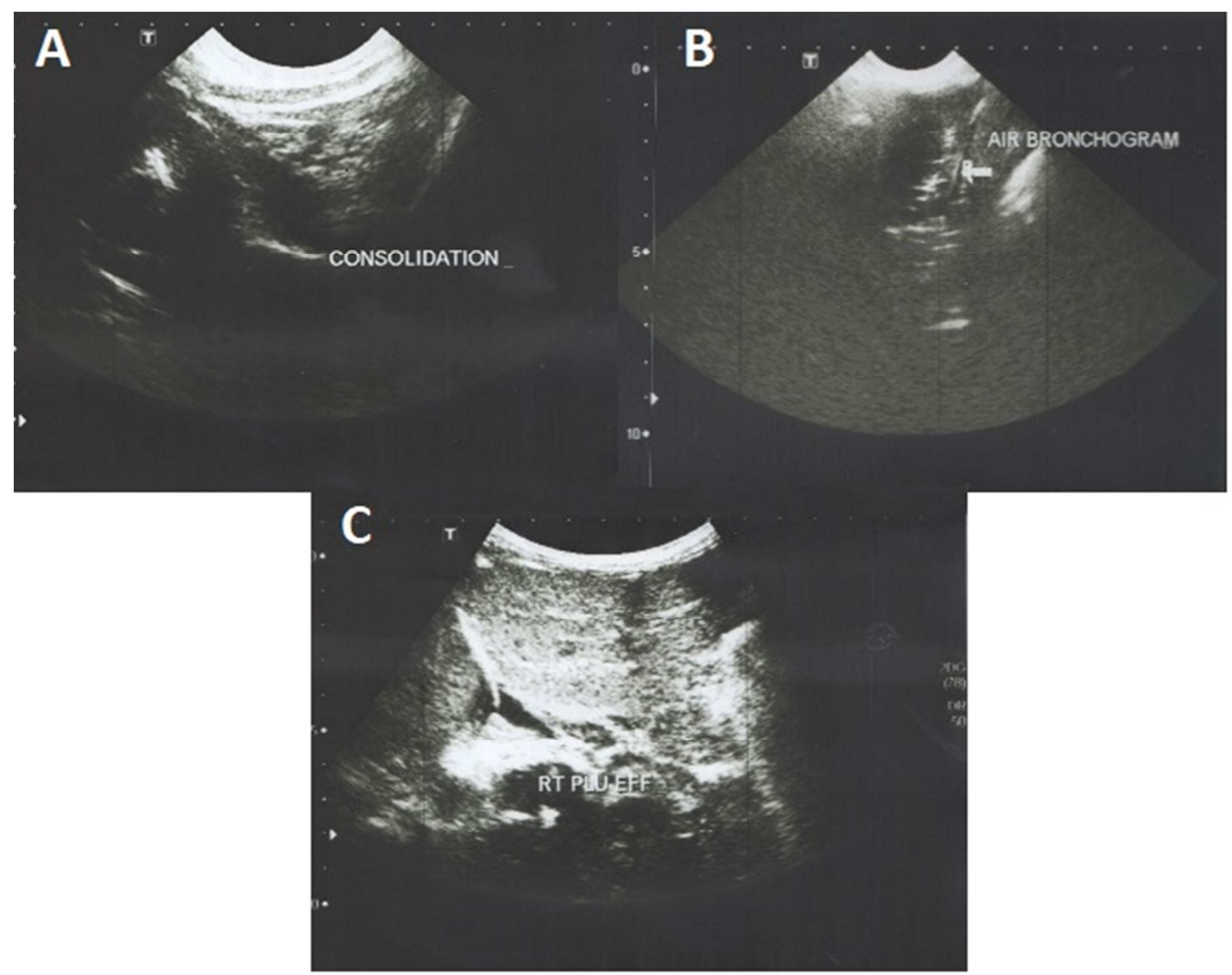

Figure 1. Lung ultrasound images showing: (A) lung consolidation in 3 years old boy; (B) air bronchogram in the same patient; (C) right pleural effusion in 4 years old girl.

The diagnostic performance of LUS in demonstration of lung involvement was calculated as follows: sensitivity of $95.6 \%$, specificity of $93.3 \%$, positive predictive value (PPV) of $97.7 \%$, negative predictive value (NPV) of $85.7 \%$ and accuracy of $94.5 \%$. [Table 4 ].

Table 4. Sensitivity, specificity, positive predictive value (PPV), negative predictive value (NPV) and accuracy of lung ultrasonography versus chest radiography in diagnosis of pneumonia.

\begin{tabular}{llllll}
\hline & Sensitivity & Specificity & PPV & NPV & Accuracy \\
\hline CXR & $88.9 \%$ & $86.7 \%$ & $95.2 \%$ & $72.2 \%$ & $86.7 \%$ \\
LUS & $95.6 \%$ & $93.3 \%$ & $97.7 \%$ & $87.5 \%$ & $94.5 \%$ \\
\hline
\end{tabular}

CXR: Chest X ray; LUS: Lung Ultrasonography.

\section{Discussion}

Chest X-ray is not routinely recommended in the diagnosis of children with community acquired pneumonia $[4,5]$. The use of lung ultrasound is actually increasing in clinical practice nowadays and although a recent international consensus conference [15] stated that lung ultrasound is a reliable method for evaluating pneumonia in adults and children, it is still recommended that when a patient needs to be assessed using an imaging technique, chest X-ray should be used first [4]. Up till now LUS is not currently included in the diagnostic work-up of pediatric CAP.

The principal finding of this study is that LUS could serve 
as a sensitive and specific method for diagnosing CAP in febrile children with respiratory distress. The sensitivity, specificity, PPV, NPV and accuracy of LUS were higher than those of CXR, so it would be reasonable to use LUS instead of CXR as the primary imaging tool to diagnose pneumonia in children, thus decreasing their exposure to ionizing radiation. Being safe, available, repeatable and inexpensive, LUS should be incorporated into a diagnostic algorithm in children with CAP.

Many studies support this finding, Urbankowska et al. showed a very high diagnostic performance of LUS (as compared to CXR) in children with suspected CAP, with sensitivity, specificity, PPV, NPV and accuracy of $93.4 \%$, $100 \%, 100 \%$ and $95.3 \%$, respectively [21]. Ultrasonography was found to be highly accurate in the diagnosis of CAP in pediatric emergency department (sensitivity and specificity of $87.1 \%$ and $94.8 \%$, respectively) [22] and also in geriatric acute care word [23]. A recently published meta-analysis of 8 studies, demonstrated very high sensitivity and specificity of LUS in diagnosis of childhood CAP (95\% and 93\% respectively) and stated that LUS is a reliable alternative imaging tool for the diagnosis of CAP in children [12]. Moreover, when using chest CT scan as a reference for diagnosis of pneumonia in the meta-analysis done by Xiong et al., LUS had a better diagnostic accuracy than CXR in adult patients with clinically suspected CAP [24].

Ultrasonography was shown to be as adequate as CT scan to detect even small amounts of pleural effusion, with sensitivity and specificity values approximating $100 \%$ [25, 26, 27]. In this study, LUS was superior to CXR in identifying parapneumonic pleural effusion in 4 patients where chest $\mathrm{x}$-ray was free. Moreover, of the 7 effusions diagnosed by CXR, 2 of which not confirmed by LUS.

Also, LUS was superior on chest $x$-ray in identifying multiple consolidations in more than one lobe $(\mathrm{P}=0.048)$. Similar results obtained by Sartori and Tombesi when LUS could detect more than one lung consolidation in $26 / 89$ patients while CXR detected the same finding only in 6/89 cases [28].

LUS is an operator-dependent technique. In this study LUS was done by an expert radiologist, however in a study conducted by Esposito et al, a pediatric resident with only 7 hours of training in LUS obtained a high sensitivity and specificity (98\% and 95\% respectively) [29]. Also recently published study by Chaves et al. demonstrated that general practitioners were prepared to perform LUS only after sevenday training. That study showed that LUS added to WHO management algorithm for acute lower respiratory infections increased the likelihood of proper pneumonia diagnosis [30]. Pedra et al. in their meta-analysis recommend training of pediatricians on LUS for diagnosis of pneumonia as this may have important implications in different clinical settings especially in resource-poor countries and small primary care clinics where CXR may not be commonly available [12]. Even if both diagnostic modalities are found in one place, Jones et al. recommend the use LUS as the initial diagnostic imaging test to evaluate children suspected of having pneumonia as this resulted in significant reduction in CXR use thus subsequently avoiding unnecessary radiation exposure, saving time and imaging coasts with no missed cases of pneumonia or significant increase in rates of adverse events [31].

The main strength of this study was the different methodology used in it which would make a valuable contribution to literature. Febrile patients with signs of respiratory distress of any severity were included and not only patients with suspected CAP as many previous studies. This was done to investigate not only the power of LUS to confirm diagnosis but also its power to exclude pneumonia in non-pneumonia patients which is actually more near to the daily clinical practice. Also bias was reduced as far as possible by using strict inclusion and exclusion criteria and by making each decision maker blinded to the information that may affect the decision. In addition it is the first study to investigate this issue among Egyptian children, a region with limited resources and high prevalence of pneumonia. This study has some limitations. First it is a single center study with small sample size. Second it is better to determine LUS sensitivity and specificity compared to the undoubtful CT scan examination; however it is unethical to perform CT scan routinely for any febrile child with respiratory distress.

\section{Conclusion}

Lung ultrasonography offers an important contribution to the diagnosis of CAP in febrile children with respiratory distress with higher sensitivity, specificity, positive and negative predictive index compared to chest X-ray. Being simple, safe, cheap and reproducible, LUS can be easily put into clinical practice in all institutions which manage children with respiratory distress.

\section{References}

[1] WHO Organization. Pneumonia. 2016. Available from: http://www.who.int/mediacentre/factsheets/fs331/en; Accessed 12 August 2017.

[2] Rudan I, Boschi-Pinto C, Biloglav Z, Mulholland K, Campbell H. Epidemiology and etiology of childhood pneumonia. Bulletin of the World Health Organization. 2008;86(5):408-16.

[3] World Health Organization: World Health Statistics 2014. Available

from: http://apps.who.int/iris/bitstream/10665/112738/1/9789240692 671_eng.pdf; Accessed 11 July 2017.

[4] Harris M, Clark J, Coote N, Fletcher P, Harnden A, McKean $\mathrm{M}$, et al. British Thoracic Society guidelines for the management of community acquired pneumonia in children: update 2011. Thorax. 2011;66(2):1-23.

[5] Bradley JS, Byington CL, Shah SS, Alverson B, Carter ER, Harrison $C$, et al. The management of community-acquired pneumonia in infants and children older than 3 months of age: clinical practice guidelines by the Pediatric Infectious Diseases Society and the Infectious Diseases Society of America. Clinical infectious diseases. 2011;53(7):25-76. 
[6] Bowen S-JM, Thomson AH. British Thoracic Society Paediatric Pneumonia Audit: a review of 3 years of data. Thorax. 2013;68(7):682-3.

[7] Davies HD, Wang EE, Manson D, Babyn P, Shuckett B. Reliability of the chest radiograph in the diagnosis of lower respiratory infections in young children. The Pediatric infectious disease journal. 1996;15(7):600-4.

[8] Brenner DJ, Hall EJ. Computed tomography-an increasing source of radiation exposure. New England Journal of Medicine. 2007;357(22):2277-84.

[9] Chotas HG, Ravin CE. Chest radiography: estimated lung volume and projected area obscured by the heart, mediastinum, and diaphragm. Radiology. 1994;193(2):403-4.

[10] Hayden GE, Wrenn KW. Chest radiograph vs. computed tomography scan in the evaluation for pneumonia. The Journal of emergency medicine. 2009;36(3):266-70.

[11] Syrjälä H, Broas M, Suramo I, Ojala A, Lähde S. Highresolution computed tomography for the diagnosis of community-acquired pneumonia. Clinical Infectious Diseases. 1998;27(2):358-63.

[12] Pereda MA, Chavez MA, Hooper-Miele CC, Gilman RH, Steinhoff MC, Ellington LE, et al. Lung ultrasound for the diagnosis of pneumonia in children: a meta-analysis. Pediatrics. 2015;135(4):714-22.

[13] Thukral BB. Problems and preferences in pediatric imaging. The Indian journal of radiology \& imaging. 2015;25(4):359.

[14] World Health Organization Pneumonia Vaccine Trial Investigators Group, Standardization of Interpretation of Chest Radiographs for the Diagnosis of Pneumonia in Children, WHO document WHO/V\&B/0135, 2001, pp. 1-32. Available at:

http://apps.who.int/iris/bitstream/10665/66956/1/WHO_V_an d_B_01.35.pdf; Accessed 8 July 2017.

[15] Volpicelli G, Elbarbary M, Blaivas M, Lichtenstein DA, Mathis G, Kirkpatrick AW, et al. International evidence-based recommendations for point-of-care lung ultrasound. Intensive care medicine. 2012;38(4):577-91.

[16] Copetti R, Cattarossi L. Ultrasound diagnosis of pneumonia in children. La radiologia medica. 2008;113(2):190-8.

[17] Lichtenstein DA, Lascols N, Mezière G, Gepner A. Ultrasound diagnosis of alveolar consolidation in the critically ill. Intensive care medicine. 2004;30(2):276-81.

[18] Reissig A, Kroegel C. Sonographic diagnosis and follow-up of pneumonia: a prospective study. Respiration. 2007;74(5):53747.

[19] Riccabona M. Ultrasound of the chest in children (mediastinum excluded). European radiology. 2008;18(2):3909.

[20] Fleiss J, Levin B, Paik M. Statistical methods for rates and proportions, 3rded. Hoboken, NJ: John Wiley \& Sons Inc; 2013.

[21] Urbankowska E, Krenke K, Drobczyński Ł, Korczyński P, Urbankowski T, Krawiec M, et al. Lung ultrasound in the diagnosis and monitoring of community acquired pneumonia in children. Respiratory medicine. 2015;109(9):1207-12.

[22] Samson F, Gorostiza I, González A, Landa M, Ruiz L, Grau M. Prospective evaluation of clinical lung ultrasonography in the diagnosis of community-acquired pneumonia in a pediatric emergency department. European Journal of Emergency Medicine. 2018;25(1):65-70.

[23] Ticinesi A1, Lauretani F, Nouvenne A, Mori G, Chiussi G, Maggio $M$, et al. Lung ultrasound and chest $\mathrm{x}$-ray for detecting pneumonia in an acute geriatric ward. Medicine (Baltimore). 2016;95(27):4153.

[24] Ye X, Xiao H, Chen B, Zhang S. Accuracy of Lung Ultrasonography versus Chest Radiography for the Diagnosis of Adult Community-Acquired Pneumonia: Review of the Literature and Meta-Analysis. PLoS ONE. 2015;10(6):0130066.

[25] Eibenberger KL, Dock WI, Ammann ME, Dorffner R, Hörmann M, Grabenwöger F. Quantification of pleural effusions: sonography versus radiography. Radiology. 1994;191(3):681-4

[26] Lichtenstein D, Goldstein I, Mourgeon E, Cluzel P, Grenier P, Rouby JJ. Comparative diagnostic performances of auscultation, chest radiography, and lung ultrasonography in acute respiratory distress syndrome. The Journal of the American Society of Anesthesiologists. 2004;100(1):9-15.

[27] Kurian J, Levin TL, Han BK, Taragin BH, Weinstein S. Comparison of ultrasound and $\mathrm{CT}$ in the evaluation of pneumonia complicated by parapneumonic effusion in children. American Journal of Roentgenology. 2009;193(6):1648-54.

[28] Sartori S, Tombesi P. Emerging roles for transthoracic ultrasonography in pulmonary diseases. World journal of radiology. 2010;2(6):203.

[29] Esposito S, Papa SS, Borzani I, Pinzani R, Giannitto C, Consonni D, et al. Performance of lung ultrasonography in children with community-acquired pneumonia. Italian journal of pediatrics. 2014;40(1):37.

[30] Chavez MA, Naithani N, Gilman RH, Tielsch JM, Khatry S, Ellington LE, et al. Agreement between the World Health Organization algorithm and lung consolidation identified using point-of-care ultrasound for the diagnosis of childhood pneumonia by general practitioners. Lung. 2015;193(4):531-8.

[31] Jones BP, Tay ET, Elikashvili I, Sanders JE, Paul AZ, Nelson BP, Spina LA, et al. Feasibility and Safety of Substituting Lung Ultrasonography for Chest Radiography When Diagnosing Pneumonia in Children. Chest. 2016; 150(1):131138. 